\title{
Opinião de alunos de psicologia sobre o ensino em avaliação psicológica
}

\author{
Alessandro Vinicius de Paula \\ Arlete Santana Pereira \\ Elizabeth do Nascimento
}

\begin{abstract}
Resumo
Entre as práticas do psicólogo há a realização de avaliação psicológica. Inadequações em avaliações psicológicas podem refletir problemas na formação do psicólogo. Este artigo apresenta uma pesquisa que investigou, por meio de um questionário, opiniões sobre avaliação psicológica, enfatizando o uso de testes psicológicos, de 358 alunos que concluíam a graduação em psicologia de quatro faculdades de Belo Horizonte, MG. Os resultados evidenciam que os alunos receberam formação na área de avaliação psicológica, porém muitos a consideraram insuficiente. Eles conhecem testes psicológicos e reconheceram que estes apresentam problemas nos parâmetros psicométricos, sendo necessária a sua melhoria. A Resolução n. ${ }^{\circ}$ 2/2003 do Conselho Federal de Psicologia, que define critérios relacionados à produção e comercialização de instrumentos psicológicos no Brasil, é desconhecida por muitos alunos. Conclui-se pela necessidade de aperfeiçoamento da formação acadêmica do psicólogo visando à aquisição de habilidades suficientes para capacitá-lo ao exercício profissional de melhor qualidade na área em questão.

Palavras-chave: Formação do psicólogo; Avaliação psicológica; Testes psicológicos.
\end{abstract}

\section{Psychology undergraduate students' opinion about psychological assessment teaching}

\begin{abstract}
Among the practical of the psychologist there is the accomplishment of psychological assessment. Mistakes in psychological assessments can reflect problems in psychologist's formation. This paper presents a research that investigated, through a questionnaire, opinions about psychological assessment, emphasizing the use of psychological tests, of 358 students who were ending the graduation in psychology from four colleges of Belo Horizonte, MG. The results evidence that the students have received formation in psychological assessment area, however many of them have considered it insufficient. They know psychological tests and have recognized that tests present problems in the psychometrics parameters, being necessary its improvement. The Resolution n. ${ }^{\circ}$ 2/2003 by Federal Council of Psychology, that defines discretions relative the production and trade of psychological tests in Brazil, is unknown by many students. We conclude that is necessary the improvement of the psychologist academic formation, aiming acquisition of abilities enough to enable him to professional exercise of better quality in the area in question.
\end{abstract}

Keywords: Psychologist's formation; Psychological assessment; Psychological tests.

\section{Introdução}

No Brasil, a Psicologia foi reconhecida oficialmente como profissão por meio da Lei n. ${ }^{\circ} 4.119$ de 27 de agosto de 1962. A partir de então, houve expansão na oferta de cursos de graduação. A psicologia é cercada de diversos desafios que podem ser relacionados, principalmente, com a formação, com a sua construção como ciência, com as suas técnicas de avaliação e de intervenção e com aspectos éticos relacionados à pesquisa e à atuação profissional.

Refletir sobre a formação dos psicólogos é relevante, uma vez que ela afeta a qualidade dos serviços prestados pelos profissionais. A formação dos psicólogos tem como principal objetivo propiciar um conjunto amplo e diversificado de conhecimentos, habilidades, atitudes e procedimentos, buscando caracterizar a psicologia como ciência e profissão.

Primi, Ladeira-Fernandez e Ziviani (2003) consideram que na formação dos psicólogos existem falhas na aquisição de conteúdos de natureza básica e na associação destes com os conteúdos de caráter mais práticos ou profissionalizantes, sendo que os psicólogos finalizam a graduação, de forma geral, evidenciando uma desvalorização do pensamento científico. Noronha e colaboradores (2002) relataram a existência da dificuldade em modificar a imagem que os alunos tinham da profissão durante a formação, bem

Endereço para correspondência:

UFMG - FAFICH - Departamento de Psicologia

Av. Presidente Antônio Carlos, 6.627 - sala 4.048 - 31270-901 - Belo Horizonte-MG

Telefone: (31) 3499-6275 - Fax: (31) 3499-4066/4068

E-mail: enasci@gold.com.br 
como a necessidade de uma avaliação sistemática sobre a qualidade da formação que tem sido oferecida nas instituições de ensino superior de psicologia no Brasil. Os problemas levantados apontam para a necessidade de discussões sobre a qualidade dos cursos de psicologia do Brasil, com estabelecimento de diretrizes curriculares sobre conteúdos básicos.

Entre as atribuições legais do psicólogo encontrase a avaliação psicológica (AP), um processo de coleta de dados que visa à identificação da queixa, ao conhecimento do sujeito e à tomada de decisões (Noronha \& Vendramini, 2003). De uma maneira geral, serve para orientar as ações profissionais do psicólogo de forma mais segura e pertinente.

Historicamente, a AP tem sido atrelada, principalmente, à utilização de testes psicológicos, que são instrumentos de uso exclusivo do psicólogo. A visão atual prevalente é de que uma AP deve ser um processo integrado, no qual se utilizam as técnicas apropriadas e necessárias ao diagnóstico (Alves, 2005; Noronha e cols., 2002; Pasquali, 2001). Assim, uma AP é um processo amplo, composto basicamente por três etapas: a) identificação de necessidades, comportamentos e processos psicológicos, podendo ser feito nesta etapa o uso de entrevista, observação, testes psicométricos e outras técnicas para a elaboração de descrições e obtenção de escores; b) integração dos dados, tanto os descritivos quanto os escores, para classificação de tipologia e de perfil; e c) formulação de hipóteses diagnósticas por meio da interpretação dos dados. Quando for o caso, a AP também inclui recomendações sobre intervenções terapêuticas que visem ao bem-estar da pessoa atendida. Portanto, além do domínio de técnicas, a AP requer do psicólogo a habilidade de compreensão e integração dos dados obtidos (Noronha, 1999). Para Quelho e colaboradores (1999), as técnicas de psicodiagnóstico permitem ao psicólogo a compreensão de fenômenos inalcançáveis de outra forma, por meio da utilização de instrumentos sofisticados e do uso de conhecimentos teóricos/práticos e daqueles oriundos de disciplinas do curso de psicologia. O valor das informações obtidas com os testes está na qualidade dos próprios instrumentos e, também, na competência científica do psicólogo (Almeida, 1999; Muñiz e cols., 2001).

As conseqüências da dificuldade que psicólogos possam ter em realizar avaliações psicológicas são o descrédito nos testes e a desconfiança de sua realidade diagnóstica (Alves, Alchieri \& Marques, 2002). Noronha e colaboradores (2002) identificam que a má formação do aluno de psicologia pode resultar em desconfiança quanto aos instrumentos de AP de forma a levá-lo à não-utilização de testes na sua atuação profissional. As autoras relacionam tal atitude com a formação técnica deficitária do corpo docente e com uma cristalização dos modelos diagnósticos, o que resulta num tratamento superficial sobre AP durante a graduação, em detrimento de uma compreensão mais ampla e profunda sobre o assunto. Andriola (1995) destaca a necessidade de treinamento e adequada formação dos psicólogos na área de psicometria, para que eles passem a ter uma visão mais abrangente e crítica quanto aos testes, não se tornando apenas aplicadores desses instrumentos. Numa pesquisa realizada por este autor (Andriola, 1999) ficou evidente a existência de deficiência na formação sobre parâmetros psicométricos, quando menos de 40\% da amostra acertou os conceitos de validade e precisão, apesar da grande maioria ter respondido corretamente a questões sobre tipos de validade e sobre padronização. Existe, portanto, a necessidade do psicólogo possuir uma sólida formação em psicometria para a adequada escolha, utilização e interpretação de resultados de testes (Alves, 2005). Noronha e colaboradores (2004) consideram que inconsistências no ensino de AP é um problema central da formação do psicólogo, relacionado a atuações profissionais impróprias.

Pesquisas internacionais também apontam para problemas na formação do psicólogo. Segundo Muñiz e colaboradores (2001), em uma pesquisa realizada com 8.690 psicólogos de países da Europa, verificou-se que, embora psicólogos europeus tenham atitude favorável em relação ao uso de testes psicológicos, a formação obtida na graduação é considerada por eles como insuficiente para o uso adequado de vários testes, o que sugere a necessidade de treinamento posterior.

Os problemas relacionados aos testes psicológicos no Brasil não se restringem à formação do psicólogo, mas abrangem a própria história da produção e utilização de testes no país. Entre as décadas de 1930 e 1960 houve intensa produção de testes, muitos autores relegando os parâmetros psicométricos a um segundo plano (Andriola, 1996), além de muitos testes serem apenas traduzidos e utilizados (Rosas, 1984). Nas décadas de 1970 e 1980, os testes psicológicos foram utilizados de forma generalizada, muitas vezes inadequada e com normas ultrapassadas. Tal realidade contribuiu para uma atitude de descrédito nos testes por parte da sociedade e críticas ao trabalho dos psicólogos. Na década de 1980, aumentaram os estudos para adaptação, construção e informatização de testes psicológicos. Houve o surgimento de cursos de especialização na área de AP (Andriola, 1996) e criação de laboratórios voltados para $\mathrm{O}$ ensino, pesquisa e desenvolvimento de instrumentos de AP (Wechsler, 2003), bem como a realização de eventos científicos específicos da área (Noronha \& Vendramini, 2003).

Para Pasquali (2001) e Wechsler (2003), no Brasil, atualmente, a área de testes psicológicos está sendo abordada de uma maneira mais científica. Mas existem dificuldades que ainda persistem:

Psico-USF, v. 12, n. 1, p. 33-43, jan./jun. 2007 
(a) a formação em um número ainda insuficiente de especialistas e pesquisadores nacionais qualificados, (b) a presença de um currículo universitário deficiente e pobre, em que as disciplinas como Psicometria, quando existem, não são administradas como tal, e sim como uma testologia (aplicação de testes) sendo, ademais, uma disciplina optativa, (c) a evasão (fuga) dos psicólogos desta disciplina, devido ao despreparo na área de disciplinas como estatística e da medida em geral, (d) apoio ainda tímido do Conselho Federal e dos Conselhos Regionais de Psicologia na área, conseqüencia da formação deficiente recebida por seus dirigentes nesta área na sua formação universitária e (e) a falta de uma maior integração efetiva entre os profissionais e pesquisadores nacionais na área [...]. (Pasquali, 2001, p. 220)

Existe atualmente uma tendência de organizações e instituições de diversos países, envolvidas na área de AP, adotarem medidas objetivando melhorar o uso de testes (Muñiz e cols., 2001). No Brasil, o Conselho Federal de Psicologia (CFP) desde 1980 vem estabelecendo uma política nacional para os instrumentos psicológicos (Noronha, Primi \& Alchieri, 2004; Pasquali, 2001). Uma ação relevante do CFP foi a promulgação da Resolução n. ${ }^{\circ}$ 2/2003, que define e regulamenta o uso, a elaboração e a comercialização de testes psicológicos no Brasil, estabelecendo que todos os testes utilizados no país devem passar por sua avaliação e aprovação. O CFP constituiu, assim, uma comissão para atuar com tal finalidade. Almeida (1999) considera relevante que a psicologia como um todo assegure minimamente a definição de parâmetros técnicos e éticos na prática de AP.

Tendo em vista os apontamentos da literatura abordada e a concepção de que a formação acadêmica compõe um dos elementos do processo ensino, aprendizagem e atuação profissional, este artigo apresenta os resultados de uma pesquisa que investigou opiniões de alunos da graduação de cursos de psicologia sobre questões relacionadas à AP. Conhecer opiniões sobre a formação na área de AP de alunos que estão concluindo cursos de psicologia é relevante, uma vez que em breve passarão a utilizar seus conhecimentos no mercado de trabalho. Os resultados do estudo podem ser relacionados à qualidade da formação e da atuação profissional e poderão oferecer elementos para o aperfeiçoamento do ensino.

\section{Método}

\section{Participantes}

A amostra desta pesquisa descritiva foi constituída por 358 alunos de psicologia que cursavam o último ano da graduação. Os alunos pertenciam a quatro instituições de Belo Horizonte, MG, sendo 20,0\% deles provenientes de uma instituição pública e 80,0\% de três instituições particulares. $\mathrm{Na}$ amostra, 39,7\% dos alunos cursavam o $9^{\circ}$ período, $54,0 \%$ cursavam o $10^{\circ}$ período e $6,1 \%$ cursavam o $11^{\circ}$ período, sendo que o curso de psicologia de três faculdades pode ser concluído regularmente em 10 períodos e de uma faculdade em 11. Em relação à idade, 59,0\% deles tinham até 24 anos, $15,0 \%$, entre 25 e 26 anos, $25,0 \%$ tinham mais de 26 anos e $1,0 \%$ não relatou a idade. Quanto ao gênero, $80,2 \%$ eram do sexo feminino $16,2 \%$ do sexo masculino, e $3,6 \%$ não informaram.

\section{Coleta de dados}

A coleta de dados ocorreu entre junho de 2004 e junho de 2005. Uma vez obtida a autorização para a realização da pesquisa junto às instituições e ao Comitê de Ética em Pesquisa, foram adotados os seguintes procedimentos com os alunos: fornecimento de informações sobre a pesquisa, bem como acerca da participação e orientações sobre a forma de preenchimento do instrumento utilizado; apresentação e assinatura do Termo de Consentimento Livre e Esclarecido, conforme Resolução n. 196 do Conselho Nacional de Saúde; preenchimento do instrumento pelo próprio aluno. Foi também disponibilizada uma lista de testes para auxiliar no preenchimento do instrumento, caso o aluno considerasse necessário.

\section{Instrumento}

Foi elaborado um questionário composto por 19 itens relativos à formação sobre AP durante a graduação, com ênfase no uso de testes. O questionário, o qual continha questões abertas e fechadas, enfatizou três eixos principais:

I. Formação acadêmica: buscou-se conhecer o número de disciplinas relacionadas à $\mathrm{AP}$ e os testes estudados;

II. Articulação entre teoria e prática na graduação: buscou-se explicitar o uso de testes psicológicos nas diversas áreas, bem como realização de AP durante a formação;

III. Identificação dos problemas mais freqüentes no uso dos testes psicológicos.

Para a análise dos dados foram utilizadas estatísticas descritivas com o objetivo de levantamento de freqüências de respostas. Ao se realizar a categorização das respostas relativas aos testes psicológicos, para alguns deles não foi possível discriminar qual versão foi estudada e/ou utilizada, uma vez que vários participantes não esclareceram este aspecto, apesar da orientação dada antes do preenchimento do questionário e do 
fornecimento de lista contendo uma relação com 170 nomes de testes psicológicos que foi confeccionada a partir de Noronha e colaboradores (2002), sendo acrescidos alguns instrumentos. Por exemplo, as Matrizes Progressivas de Raven podem ser encontradas nas versões colorida, geral e avançada. No entanto, em algumas respostas havia apenas uma menção genérica do nome do teste.

\section{Apresentação e discussão dos resultados}

Formação acadêmica em avaliação psicológica

Com base na estrutura curricular atual, as faculdades investigadas oferecem de três a quatro disciplinas obrigatórias na área de AP e as disciplinas optativas mais cursadas pelos alunos foram: Rorschach
(12,1\%); Técnicas Gráficas (10,1\%); Psicodiagnóstico Miocinético - PMK (6,9\%) e Teste de Apercepção Temática/Teste de Apercepção Infantil - TAT/CAT $(4,2 \%)$. Duas faculdades não oferecem disciplinas optativas na área e, quando outras chegam a ofertar esta modalidade de disciplina, um percentual baixo de alunos chegou a cursá-las. Tal realidade pode estar expressando desinteresse dos alunos pelo assunto e/ou dificuldade das instituições de ensino para fomentar o interesse.

Foi solicitado aos alunos que citassem os testes psicológicos estudados na graduação. A Tabela 1 apresenta os testes citados, em ordem decrescente de citação. Solicitou-se também que os alunos citassem os testes psicológicos utilizados nos principais estágios da graduação (Tabela 2).

Tabela 1 - Testes estudados durante a graduação

\begin{tabular}{lcc}
\hline \multicolumn{1}{c}{ Nome do teste } & Número de citações & Freqüência (\%) \\
\hline WISC (Escala Wechsler de inteligência p/ crianças) & 259 & 72,7 \\
DFH (Desenho da Figura Humana) & 204 & 79,7 \\
Matrizes Progressivas de Raven & 194 & 54,5 \\
BPR - 5 (Bateria de Prova de Raciocínio) & 177 & 49,8 \\
HTP: Casa, árvore e pessoa & 165 & 46,3 \\
Teste do Desenho Wartegg & 162 & 45,5 \\
IFP (Inventário Fatorial de Personalidade) & 159 & 44,7 \\
G 36 (Teste de Inteligência Não-Verbal) & 159 & 44,7 \\
Bender & 150 & 42,1 \\
Rorschach & 136 & 38,2 \\
Teste das Fábulas & 116 & 32,6 \\
R 2 (Teste Não-Verbal de Inteligência p/ crianças) & 115 & 32,3 \\
R 1 (Teste Não-Verbal de Inteligência) & 100 & 28,1 \\
Figuras Complexas de Rey & 95 & 26,7 \\
D 2 (Teste de Atenção Concentrada) & 93 & 26,1 \\
\hline Número de respondentes & 356 & 100,0 \\
\hline
\end{tabular}


Tabela 2 - Testes utilizados nos principais estágios

\begin{tabular}{lcc}
\hline \multicolumn{1}{c}{ Nome do teste } & Número de citações & Freqüência (\%) \\
\hline HTP (Casa, árvore, pessoa)/HTPF & 75 & 20,9 \\
Matrizes Progressivas de Raven & 65 & 18,1 \\
WISC III (Escala Wechsler de inteligência p/ crianças) & 61 & 17,0 \\
Bender & 60 & 16,8 \\
Teste das Fábulas & 51 & 14,2 \\
PMK (Psicodiagnóstico miocinético) & 43 & 12,0 \\
Teste do Desenho Wartegg & 39 & 10,9 \\
DFH (Desenho da Figura Humana) & 38 & 10,6 \\
G 36 (Teste de Inteligência Não-Verbal) & 38 & 10,6 \\
IFP (Inventário Fatorial de Personalidade) & 38 & 10,6 \\
D 2 (Teste de Atenção Concentrada) & 30 & 8,4 \\
R 1 (Teste Não-Verbal de Inteligência) & 28 & 7,8 \\
Teste AC & 19 & 5,3 \\
IAT: Inventário de Atitude de Trabalho & 14 & 3,9 \\
\hline Número de respondentes & 358 & - \\
\hline
\end{tabular}

Pode-se considerar que a freqüência de citação de testes objetivos e projetivos é equivalente, com prevalência de testes que se propõem a mensurar inteligência e personalidade, sendo que testes objetivos tiveram pouca expressão na avaliação da personalidade. Nos estágios, que eram de vários tipos (curricular, extracurricular e outros), houve maior variabilidade de instrumentos utilizados.

Alves e colaboradores (2002) realizaram um estudo com o objetivo de conhecer a situação do ensino das Técnicas de Exame Psicológico segundo os professores da área. $\mathrm{Na}$ opinião dos professores, os testes TAT, WISC, Matrizes Progressivas de Raven, Rorschach, HTP e Bender são indispensáveis para a atuação profissional competente. Para uma formação mais completa deveriam ser incluídos PMK, Escala Wechsler de Inteligência para Adultos (WAIS), Wartegg, Columbia, Goodenough-Harris e DFH de Machover, além de um inventário de interesse e uma bateria de aptidão. $\mathrm{Na}$ presente pesquisa, a utilização de testes nos diversos tipos de estágios contemplou, parcialmente, a relação de testes indicados como indispensáveis no estudo de Alves e colaboradores (2002), não tendo sido mencionados o Rorschach e o TAT. Dos outros que complementam a relação, apenas o Wartegg, duas baterias de aptidão (BPR5 e D-2) e um inventário de interesse (QVI) foram citados.

Diante de tais informações, pode-se identificar alguma discrepância entre a variabilidade de instrumentos utilizados no mercado de trabalho e o que é ensinado ou considerado relevante pelos professores da área de AP. No entanto, cabe destacar que não foi feito um levantamento dos testes ensinados aos alunos participantes da pesquisa Psico-USF, v. 12, n. 1, p. 33-43, jan./jun. 2007 pelas faculdades. Assim, pode ser que algum teste ensinado não tenha sido lembrado e citado pelos alunos, embora os participantes pudessem ter acesso a uma lista de testes durante o preenchimento do questionário.

No que se refere à necessidade de todos os testes utilizados no país serem avaliados e aprovados pelo CFP, segundo a Resolução n. ${ }^{\circ}$ 2/2003, verificou-se que a maioria dos testes da relação obtida por Alves e colaboradores (2002) já recebeu parecer favorável, ${ }^{1}$ exceto o Wartegg e o Goodenough-Harris. No caso dos testes utilizados pela amostra em questão, os Wartegg e IAT receberam parecer desfavorável pelo CFP, enquanto o QVI não foi enviado ao CFP para avaliação até a conclusão da presente pesquisa.

No estudo de Noronha e colaboradores (2002) que contemplou a análise dos testes mais citados pelos alunos de psicologia, verifica-se que 40,0\% dos instrumentos não apresentavam normatização brasileira (WISC, PMK, Raven - Escala Geral, CAT - Animal e Humano, Rorschach, Inventário de Interesse de Angelini e Thurstone, HTP e TAT). Atualmente, 66,0\% destes instrumentos receberam parecer favorável do CFP. De acordo com os critérios do CFP, normatização, padronização, validade e precisão dos testes são requisitos necessários para a sua aprovação. Com isso, verifica-se que aumentou o número de testes que passou a atender minimamente aos critérios científicos. Esta constatação pode estar expressando um avanço na área de AP, uma vez que os principais testes psicológicos objetivos não apresentavam adaptação e normatização brasileira (Hutz \& Bandeira, 1993) e que o estudo de Noronha e Vendramini 
(2003) evidenciou que alguns testes, tanto de inteligência quanto de personalidade, mas principalmente estes últimos, careciam de padronização, validação e precisão, sendo constatado crescimento na realização de estudos acerca dos três parâmetros.
Verificaram-se também os aspectos positivos e negativos que os alunos identificavam na sua formação em AP. O processo de categorização das respostas evidenciou que existe relação entre o que os alunos consideraram positivo e negativo, conforme está exposto na Tabela 3.

Tabela 3 - Aspectos positivos e negativos da formação acadêmica na área de AP

\begin{tabular}{lcc}
\multicolumn{1}{c}{ Aspectos da formação acadêmica na área de AP } & $\begin{array}{c}\text { Número de } \\
\text { citações }\end{array}$ & $\begin{array}{c}\text { Freqüência } \\
(\%)\end{array}$ \\
\hline \multicolumn{1}{c}{ Aspectos positivos } & 111 & 24,5 \\
Capacitação profissional dos alunos. & 64 & 14,1 \\
Conhecimento sobre testes/outras técnicas de AP. & 27 & 5,6 \\
Qualidade dos professores. & 16 & 3,5 \\
Qualidade das disciplinas. & 11 & 2,4 \\
Oportunidades de realização de estágios na área de AP. & 108 & 21,0 \\
Insuficiência no conteúdo. & 60 & 11,7 \\
Insuficiência no número de disciplinas ofertadas. & 41 & 8,0 \\
Insuficiência de material didático. & 32 & 6,2 \\
Baixa qualidade técnica dos professores. & 30 \\
Dificuldade de articulação entre teoria e prática do conteúdo das disciplinas da & 28 \\
área de AP. & 3,8 \\
Disciplinas não contribuem para uma capacitação profissional suficiente. & 5,6 \\
\hline
\end{tabular}

As outras técnicas psicológicas, além de testes, que os alunos consideram importantes para a realização de uma AP foram: entrevista (39,1\%), dinâmica (12,6\%), anamnese $(12,4 \%)$, técnicas lúdicas $(6,6 \%)$ e observação do comportamento (6,3\%). Outros recursos técnicos foram citados por poucos estudantes, tais como questionários $(1,3 \%)$, provas situacionais $(1,2 \%)$ e técnicas gráficas diversas $(1,2 \%)$. Destaca-se que a entrevista é a técnica considerada mais importante pelos pesquisados. Ao percentual obtido pela técnica entrevista é possível acrescentar o obtido pela técnica anamnese, o que resultaria em 51,5\% de freqüência de citação, uma vez que Tavares (2000) classifica a anamnese como uma das finalidades de uma entrevista.

Barbosa e Nascimento (2003) empreenderam um estudo que teve como objetivo caracterizar como os profissionais realizam AP e que contou com uma amostra de 105 psicólogos residentes na região metropolitana de Belo Horizonte. Os $(63,2 \%)$ que incluem regularmente o uso de testes psicológicos na AP também utilizam entrevista (69,5\%). Os demais psicólogos (48,6\%), cujas avaliações psicológicas não incluem testes, apontaram a entrevista (81,1\%) como principal técnica utilizada. Pode-se concluir que o psicólogo em formação valoriza a entrevista e que outras técnicas, além de testes, também são consideradas relevantes. Porém, ao confrontarmos esse dado com o estudo de Barbosa e Nascimento (2003), percebe-se que o profissional enfatiza, substancialmente, a utilização de entrevistas.

Foi solicitado aos alunos que citassem a AP realizada por eles que contemplou o maior número de etapas. Diante dessa informação, verificou-se que existe a tendência de o próprio aluno realizar a maioria das etapas da AP (entrevista, aplicação de técnicas psicológicas, correção e interpretação de resultados). Outra tendência identificada foi a de o aluno receber auxílio mais freqüentemente para a correção e interpretação dos resultados e para a entrevista de devolução. Além disso, a elaboração do relatório psicológico é a etapa realizada com menor freqüência pelo aluno, com indicativos de ser executada somente por terceiros ou simplesmente negligenciada.

Pasquali (2001) salienta a necessidade de se realizar a AP de forma integrada para diversas finalidades (diagnóstico, avaliação para modificação na intervenção terapêutica, etc.) e Noronha (1999) descreve a importância de o profissional desenvolver habilidade suficiente para fazer relações entre os resultados obtidos por meio de testes e outras técnicas utilizadas na AP. Os alunos de psicologia participantes da amostra tenderam a realizar a maioria das etapas da AP, o que exigiu deles conhecimento suficiente 
para conduzir entrevistas, selecionar e aplicar instrumentos psicológicos, bem como outras técnicas de AP. É oferecido a eles maior suporte para a correção e interpretação de resultados. A elaboração de relatórios psicológicos, importante para a comunicação dos resultados de uma AP, e freqüentemente requisitado ao profissional em diversas áreas de atuação, é pouco privilegiada na formação. Assim, parece haver uma tendência de se realizar a AP de forma incompleta, limitando as oportunidades de o aluno treinar a integração dos dados obtidos durante o processo de AP.

Os participantes também prestaram informações sobre os estágios considerados por eles como sendo os mais relevantes na formação acadêmica. As áreas mais citadas foram psicologia clínica $(32,0 \%)$, seguida da psicologia organizacional/trabalho (20,5\%), psicologia escolar (7,7\%) e psicologia social/comunitária (7,6\%). Outros estágios, menos freqüentes, foram nas áreas de psicologia hospitalar (2,5\%), psicologia do esporte $(3,0 \%)$, técnica de exame psicológico $(2,2 \%)$, psicologia jurídica $(1,8 \%)$, psicologia do desenvolvimento humano $(1,7 \%)$, orientação profissional $(0,9 \%)$ e saúde mental $(0,7 \%)$.

A clínica é a área mais prevalente nos principais estágios realizados pelos participantes, o que está de acordo com uma tendência identificada na psicologia por vários autores, como Andriola (1996), Branco (1998), Bock (1999), Assunção (1999), Pasquali (2001) e Muñiz e colaboradores (2001). A prevalência da clínica pode ser compreendida como uma manutenção do modelo clínico da medicina, uma das áreas de conhecimento onde a psicologia tem suas raízes (Assunção, 1999; Bock, 1999; Branco, 1998), o que, para alguns autores, seria negativo para o desenvolvimento da psicologia (Andriola, 1996; Pasquali, 2001). Por outro lado, com base nos estágios relatados pela amostra, identifica-se que várias outras áreas nas quais a atuação do psicólogo é necessária vêm se solidificando.

Entre os estágios considerados os mais relevantes, em 46,0\% deles não houve a utilização de testes, em 37,7\% eles foram utilizados e em 16,3\% não ficou esclarecido se esse tipo de técnica foi utilizada. As principais justificativas para a não-utilização de testes nos estágios foram: ausência de necessidade (22,1\%); a abordagem de referência do aluno não privilegia o uso de testes $(7,2 \%)$ e utilização de outras técnicas ou recursos $(5,7 \%)$.

Os participantes foram indagados sobre os principais problemas ${ }^{2}$ relacionados à utilização dos testes no Brasil. Os problemas estão apresentados na Tabela 4.

Tabela 4 - Problemas relacionados à utilização de testes psicológicos no Brasil

\begin{tabular}{|c|c|c|c|}
\hline Categorias & Problemas & $\begin{array}{l}\text { Número de } \\
\text { citações }\end{array}$ & $\begin{array}{l}\text { Freqüência } \\
(\%)\end{array}$ \\
\hline \multirow{2}{*}{ Formação } & Formação e treinamento insuficiente para uso de testes. & 151 & 40,5 \\
\hline & Superficialidade no ensino de testes. & 08 & 2,0 \\
\hline \multirow{4}{*}{ Uso } & $\begin{array}{l}\text { Realização de AP incompleta ou com problema em } \\
\text { alguma etapa. }\end{array}$ & 60 & 15,0 \\
\hline & Realização de AP baseada exclusivamente em testes. & 45 & 11,3 \\
\hline & Custo elevado dos testes. & 25 & 6,1 \\
\hline & Utilização de fotocópias de testes. & 15 & 3,8 \\
\hline \multirow{3}{*}{ Instrumento } & Inadequação e/ou ausência de normatização brasileira. & 115 & 26,3 \\
\hline & Inadequação e/ou ausência de validação. & 89 & 20,8 \\
\hline & Pesquisas insuficientes. & 26 & 6,2 \\
\hline \multirow{4}{*}{ Éticos } & Utilização abusiva e/ou inadequada dos testes. & 80 & 20,0 \\
\hline & Utilização de testes por outros profissionais. & 63 & 15,5 \\
\hline & Divulgação indevida de testes. & 24 & 6,0 \\
\hline & Fiscalização insuficiente por parte do CFP. & 10 & 2,5 \\
\hline Epistemológicos & Descrédito na utilidade de testes psicológicos. & 08 & 2,0 \\
\hline
\end{tabular}

Entre os problemas relacionados à utilização de testes psicológicos no Brasil destaca-se que 40,5\% da amostra citaram a insuficiência do treinamento dos psicólogos para uso de testes psicológicos, o que está de acordo com opiniões de psicólogos investigados por Muñiz e colaboradores (2001). A insuficiência do treinamento torna-se evidente nos problemas relacionados à realização de AP de forma incompleta ou baseada exclusivamente em testes. A graduação deveria fornecer, por excelência, as principais oportunidades para os alunos obterem conhecimentos e habilidades para a utilização de testes psicológicos. Possibilidades secundárias para o incremento dos conhecimentos seriam, por exemplo, cursos diversos como de 
aperfeiçoamento e de atualização. Outros problemas importantes estão relacionados à normatização e à validade dos instrumentos. O problema possivelmente está sendo reduzido com a Resolução n. ${ }^{\circ}$ 2/2003, conforme discutido anteriormente.

Entre os alunos pesquisados, 58,9\% não se consideraram aptos para planejar e executar avaliações psicológicas fazendo uso de testes psicológicos, alegando, principalmente, que a formação acadêmica foi insuficiente $(35,2 \%)$ e que não apresentam interesse pela área $(12,3 \%)$. Os outros $41,1 \%$ consideram-se aptos para realizar tal atividade, alguns argumentando que o conhecimento adquirido em sua formação foi suficiente $(15,5 \%)$, alguns considerando que necessitarão de supervisão $(3,4 \%)$ e também que as suas experiências extra-acadêmicas ajudaram na capacitação para a realização da tarefa $(3,1 \%)$. Destaca-se, contudo, que $69,6 \%$ consideraram importante a realização de AP na prática profissional, pois tal atividade favorece $\mathrm{O}$ tratamento/intervenção $(13,4 \%)$, oferece suporte e confrabilidade ao trabalho do psicólogo $(5,0 \%)$ e é utilizada em diversas áreas de atuação profissional (3,9\%). Os alunos que não consideraram importante realizar AP $(28,2 \%)$ argumentaram, principalmente, que preferem outras formas de intervenção $(4,2 \%)$ ou que a abordagem teórica com a qual se identificam dispensaria o uso de testes $(3,9 \%)$.

Dos alunos de graduação em psicologia que compuseram a amostra de Andriola e Lima (1999), $92,1 \%$ consideraram importante o uso de testes psicológicos e 73,7\% manifestaram a intenção de trabalhar com testes psicológicos. No estudo de Noronha (1999), apenas 43,0\% dos psicólogos utilizavam testes na atuação profissional. Dos que não utilizam testes em suas atividades profissionais, alguns deles nunca fizeram uso de testes. Barbosa e Nascimento (2003) relataram que, dos psicólogos que realizam AP com pouco ou nenhum uso de testes psicológicos, 67,6\% já fizeram uso desses instrumentos no passado, abandonando a prática por considerarem que os testes são pouco adequados e que há incompatibilidade entre eles e a abordagem teórica adotada pelo profissional.

A maioria dos alunos participantes do presente estudo (69,6\%) considerou importante a utilização de AP, apesar de apenas $41,1 \%$ se considerarem aptos para a realização da atividade ao final da graduação; a maioria da amostra de alunos pesquisados por Andriola e Lima (1999) também manifestou ser favorável ao uso de testes psicológicos. Ao se comparar estes dados com os resultados de Noronha (1999) e os de Barbosa e Nascimento (2003), parece haver um declínio na importância que se confere ao uso de testes na AP, havendo até mesmo um abandono da sua utilização. Tal constatação nos remete a novas questões para futuras investigações. Noronha e colaboradores (2002) apontam a existência de correlação entre a má qualidade na formação e a não-utilização de testes. Deficiência no entendimento de conteúdos relacionados a parâmetros psicométricos, como validade e precisão, foi detectada por Andriola e Lima (1999) entre alunos da graduação. Estes últimos autores consideram que uma melhor preparação dos estudantes para utilizar testes psicológicos de maneira ética, competente e responsável é necessária. Assim, é possível que a formação insuficiente do psicólogo para o uso de testes psicológicos acabe por influenciar a sua atitude de desvalorizar ou abandonar estes instrumentos de AP na sua prática profissional.

Foi verificada a principal linha teórica com a qual o aluno se identifica. As mais citadas foram: psicanálise (36,6\%), psicologia existencial/humanista/ fenomenológica $^{3}(24,9 \%)$, psicologia comportamental (8,1\%), gestalt/gestaltterapia $(5,3 \%)$ e psicologia sistêmica (3,6\%). Poucos alunos $(1,4 \%)$ não se identificam com nenhuma linha teórica. Pasquali (2001) indica que a desvalorização da psicometria no Brasil até a década de 1980 foi influenciada pela proeminência de enfoques humanistas e dialéticos na compreensão do comportamento humano, que constituíam forma de reação ao behaviorismo americano. Parece, portanto, que, nas faculdades investigadas, aqueles enfoques proeminentes da década de 1980 ainda estão bastante presentes.

Quanto ao conhecimento da Resolução n. ${ }^{\circ}$ 2/2003 do CFP, o estudo indicou que a maioria dos alunos $(83,0 \%)$ desconhece a resolução, que poucos $(12,0 \%)$ demonstraram conhecê-la e alguns $(5,0 \%)$ não se manifestaram sobre o assunto. $\mathrm{O}$ item relacionado ao assunto foi construído de forma que era possível checar se o aluno realmente conhecia o conteúdo da resolução supracitada. No entanto, ao responder a outras questões, alguns alunos, além daqueles que demonstraram conhecer a resolução, manifestaram preocupação acerca do fato de alguns testes não terem sido aprovados pelo CFP.

\section{Conclusões}

Esta pesquisa descritiva buscou identificar as opiniões de uma amostra de alunos da capital mineira, que estavam concluindo o curso de psicologia, quanto a vários aspectos relacionados com a formação acadêmica em avaliação psicológica (AP). De forma geral, os alunos receberam formação na área de AP. Os conteúdos abordados nas disciplinas não foram 
investigados neste estudo, mas $21,0 \%$ da amostra considerou que há insuficiência de conteúdos na área de AP, e o treinamento dos psicólogos para uso de testes é insuficiente segundo 40,5\% dos respondentes. Além do abordado nas disciplinas obrigatórias, poucos alunos cursaram outras disciplinas relacionadas a testes psicológicos. $\mathrm{Na}$ graduação os estudantes têm oportunidade de conhecer, nas disciplinas e nos estágios, os testes considerados como repertório mínimo necessário para a formação de psicólogos. Nos estágios, atividades acadêmicas articuladas com o mercado de trabalho, ocorreu a utilização de maior diversidade de testes, o que pode ter exigido do aluno o conhecimento de um maior número de instrumentos.

Os alunos identificaram vários problemas tanto na sua formação quanto na utilização de testes no Brasil. Muitos consideraram que a academia não os capacita o suficiente para realizarem AP e que os próprios testes disponíveis no país apresentam problemas relacionados, principalmente, à padronização e à normatização. A formação na área de AP apresenta deficiências, identificadas pelos próprios alunos, que parecem afetar a futura atuação profissional do psicólogo. Outra conclusão, obtida pela identificação de problemas relacionados à utilização de testes no Brasil, diz respeito à necessidade da melhoria na qualidade dos testes comercializados no país, principalmente no que se refere aos seus parâmetros psicométricos.

As duas conclusões citadas anteriormente remetem a uma terceira, mais favorável. Os alunos foram capazes de identificar diversos fatores que afetam sua formação acadêmica, bem como os que afetam a utilização de testes psicológicos, o que pode estar expressando mudanças quanto a eles apresentarem uma visão crítica sobre o assunto.

Neste contexto de mudanças destaca-se que a Resolução n. ${ }^{\circ}$ 2/2003 do Conselho Federal de Psicologia (CFP) tem mostrado ser um marco na definição de critérios relacionados à produção e comercialização de instrumentos psicológicos no Brasil. Uma vez aplicados os critérios científicos para que os testes possam ser comercializados, infere-se a existência de condições minimamente aceitáveis de qualidade dos instrumentos. $\mathrm{O}$ incremento de pesquisas e a melhoria na qualidade dos serviços prestados pelos psicólogos à sociedade constituem duas conseqüências esperadas depois da implementação da resolução.

É digno de nota o alto grau de desconhecimento da Resolução n. ${ }^{\circ} 2 / 2003$ pelos participantes da pesquisa. Eles apresentam a peculiaridade de estar concluindo a formação acadêmica, portanto, em pouco tempo serão egressos dos cursos de psicologia, os quais terão como desafio a sua inserção no mercado de trabalho. As dissonâncias verificadas entre a formação dos psicólogos e demandas da atualidade, como a política do CFP, podem trazer implicações negativas para o futuro exercício profissional.

Apesar desta pesquisa estar limitada a uma única cidade do país, os seus resultados sugerem a necessidade de estudos e reflexões sobre a formação acadêmica dos psicólogos, uma vez que a aquisição de conhecimentos, habilidades e atitudes no âmbito da academia é necessária e favorável tanto para o desempenho profissional quanto para o próprio desenvolvimento da psicologia como ciência e profissão. Por fim, ampliar a pesquisa para amostras de outras localidades e regiões do Brasil poderá fornecer informações que complementem o entendimento das questões levantadas pelo presente estudo. Recomenda-se também que novos estudos possam ser conduzidos com o objetivo de aprofundar a investigação sobre, por exemplo, as possíveis repercussões da Resolução n. ${ }^{\circ}$ 2/2003 na prática profissional.

\section{Notas}

${ }^{1}$ De acordo com lista de testes com parecer favorável pelo Conselho Federal de Psicologia, atualizada em dezembro de 2005. Disponível em <www.pol.org.br>, consultada em 7 de fevereiro de 2006

2 Para a análise dos resultados da pesquisa, os problemas na utilização dos testes foram divididos em problemas de formação, problemas de uso, problemas do instrumento, problemas éticos e problemas epistemológicos. O mesmo recurso metodológico foi utilizado por Noronha (1999).

${ }^{3}$ Nesta pesquisa não foi possível separar as linhas teóricas psicologia existencial, humanista e fenomenológica - em virtude de os participantes citarem-nas de forma inconsistente, juntas ou separadamente.

\section{Referências}

Almeida, L. S. (1999). Avaliação psicológica: exigências e desenvolvimentos nos seus métodos. Em S. M. Wechsler \& R. S. L. Guzzo (Orgs.). Avaliação psicológica: perspectiva internacional (pp. 41-55). São Paulo: Casa do Psicólogo.

Alves, I. C. B. (2005). A banalização de diagnósticos. Psicologia: Ciência e Profissão (Diálogos), 2(3), 47-49.

Alves, I. C. B., Alchieri, J. C. \& Marques, K. C. (2002). As técnicas de exame psicológico ensinadas nos cursos de graduação de acordo com os professores. Psico-USF, 7(1), 77-88.

Andriola, W. B. (1995). Problemas e perspectivas quanto ao uso dos testes psicológicos do Brasil. Psique, 6, 46-57. 
Andriola, W. B. (1996). Avaliação psicológica no Brasil: considerações a respeito da formação dos psicólogos e dos instrumentos utilizados. Psique, 8, 98-108.

Andriola, W. B. \& Lima, M. G. O. (1999). A avaliação da opinião e do grau de conhecimento de estudantes de psicologia sobre os testes psicológicos: o caso da Universidade Federal do Ceará (UFC). Psique, 9, 91106.

Assunção, M. M. S. (1999). Curso de psicologia: algumas reflexões sobre o bacharelado e a licenciatura. Educação em Revista, Belo Horizonte, 29, 45-60.

Barbosa, M. A. \& Nascimento, E. (2003). Prática da avaliação psicológica: um estudo descritivo. Trabalho apresentado na XXXIII Reunião Anual da Sociedade Brasileira de Psicologia, Belo Horizonte, MG.

Bock, A. M. B. (1999). A psicologia a caminho do novo século: identidade profissional e compromisso social. Estudos de Psicologia, 4(2), 315-329.

Branco, M. T. C. (1998). Que profissional queremos formar? Psicologia Ciência e Profissão, 18(3), 28-35.

Conselho Federal de Psicologia (2003). Resolução $n^{\circ}$ 2/2003. Disponível em: <www.pol.org.br>. Acesso em: 25 set. 2003.

Conselho Federal de Psicologia (2006). Lista de testes com parecer favorável. Disponível em: <www.pol.org.br>. Acesso em: 7 fev. 2006.

Hutz, C. S. \& Bandeira, D. R. (1993). Tendências contemporâneas no uso de testes: uma análise da literatura brasileira e internacional. Psicologia: Reflexão e Crítica, 6(1/2), 85-101.

Muñiz, J., Bartran, D., Evers, A., Baben, D., Matesic, K., Glabeke, K., Fernández-Hermida, J. R. \& Zaal, J. N. (2001). Testing practices in European countries. European Journal of Psychological Assessment, 17(3), 201211.

Noronha, A. P. P. (1999). Avaliação psicológica segundo psicólogos: usos e problemas com ênfase nos testes. (Tese de Doutorado em Psicologia). Campinas: PUC Instituto de Psicologia e Fonoaudiologia.

Noronha, A. P. P, Baldo, C. R., Almeida, M. C., Freitas, J. V., Barbin, P. F. \& Cozoli, J. (2004). Conhecimento de estudantes a respeito de conceitos de avaliação psicológica. Psicologia em Estudo, 9(2), 263-269.

Noronha, A. P. P., Oliveira, A. F., Cobêro, C.; Paula, L. M., Cantalice, L. M., Guerra, P. B. C., Martins, R. M. M. \& Felizatti, R. (2002). Instrumentos psicológicos mais conhecidos por estudantes do sul de Minas Gerais. Avaliação Psicológica - IBAP, 1(2), 151-158.

Noronha, A. P. P. \& Vendramini, C. M. M. (2003). Parâmetros psicométricos: estudo comparativo entre testes de inteligência e de personalidade. Psicologia: Reflexão e Crítica, 16(1), 177-182.

Noronha, A. P. P., Primi, R. \& Alchieri, J. C. (2004). Parâmetros psicométricos: uma análise de testes psicológicos comercializados no Brasil. Psicologia Ciência e Profissão, 24(4), 88-99.

Pasquali, L. (Org.). (2001). Técnicas de Exame PsicológicoTEP: manual. São Paulo: Casa do Psicólogo; Conselho Federal de Psicologia.

Primi, R., Ladeira-Fernandez, J. \& Ziviani, C. (2003). O Provão de psicologia: objetivos, problemas, conseqüências e sugestões. Psicologia: Teoria e Pesquisa, 19(2), 109-116.

Quelho, A. M. C., Munhoz, A. M. H., Damião, J. B. B. \& Gomes, L. M. B. (1999). Repensando a supervisão em psicodiagnóstico: a relação teórica e prática uma questão de ensino e/ou aprendizagem. PsicoUSF, 4(2), 13-22.

Rosas, P. (1984). A psicologia na Revista Brasileira de Estudos Pedagógicos. Revista Brasileira de Estudos Pedagógicos, 65(150), 314-336.

Tavares, M. A entrevista clínica (2000). Em Cunha, J. A. Psicodiagnóstico $V$ - 5 a edição revisada e ampliada (pp. 4556). Porto Alegre: Artes Médicas Sul.

Wechsler, S. M. (2003). Test development and use in Brazil: its history and current status. Testing International, 13(2), 11-12.

Recebido em maio de 2006 Reformulado em março de 2007 Aprovado em abril de 2007 
Sobre os autores:

Alessandro Vinicius de Paula é psicólogo e mestrando em Psicologia (linha de concentração: Psicologia Social) pela Universidade Federal de Minas Gerais, professor substituto da Universidade Federal de Minas Gerais e pesquisador nas áreas de Psicologia do Trabalho e Avaliação Psicológica.

Arlete Santana Pereira é psicóloga e mestre em Psicologia (linha de concentração: Desenvolvimento Humano) pela Universidade Federal de Minas Gerais, professora de Métodos e Técnicas de Pesquisa em Psicologia do Centro Universitário Newton Paiva e pesquisadora nas áreas de Avaliação Psicológica e Educação Infantil.

Elizabeth do Nascimento é doutora em Psicologia pela Universidade de Brasília, psicóloga e mestre em Psicologia Social pela Universidade Federal de Minas Gerais, professora de Técnicas de Exame Psicológico no Departamento de Psicologia da Universidade Federal de Minas Gerais, professora do programa de Pós-Graduação em Psicologia da Universidade Federal de Minas Gerais, pesquisadora e membro do Laboratório de Avaliação das Diferenças Individuais (LADI/UFMG). 
